\title{
Kompaktes, infrarotoptisches Zweikanal - Gasmesssystem zur Überwachung von Kohlendioxid in Zellinkubatoren
}

\author{
J. Huber ${ }^{(1)}$, A. Eberhardt ${ }^{(1)}$, A. Müller ${ }^{(2)}$, J. Wöllenstein ${ }^{(2)}$ \\ (1) Fraunhofer-Institut für Physikalische Messtechnik IPM, Heidenhofstr. 8, 79110 Freiburg; \\ jochen.huber@ipm.fraunhofer.de \\ (2) Institut für Mikrosystemtechnik IMTEK, Technische Fakultät der Universität Freiburg, \\ Georges-Köhler-Allee 102, 79110 Freiburg
}

\section{Kurzfassung}

Es wird ein Messsystem für die Überwachung der Kohlendioxidkonzentration in Zellinkubatoren vorgestellt. Das System arbeitet mit dem Prinzip eines Zweikanalfilterfotometers und detektiert $\mathrm{CO}_{2}$ durch molekülspezifische Infrarotabsorption. Das System arbeitet im Messbereich von $0-10 \% \mathrm{CO}_{2}$. Die Zweikanalmessmethode mit Mess- und Referenzkanal reduziert Drifteinflüsse und erhöht die Stabilität des Messsystems. Die Ansteuerung der Komponenten, sowie die analoge und digitale Signalverarbeitung der Messdaten wurden sensornah integriert. Durch die zeitnahe Überwachung der Kohlendioxidkonzentration kann der $\mathrm{pH}$-Wert innerhalb eines Inkubators auf optimale Zellwachstumsbedingungen geregelt werden. Zur Zeit sind keine kostengünstigen Systeme am Markt erhältlich. Das vorgestellte System mit einfachen Infrarotkomponenten erfüllt die Anforderungen und ist günstig zu realisieren.

\section{Einleitung}

Zellen, als kleinste lebende Einheiten eines Organismus, sind in der Biologie die Grundlage für viele spezifische, biochemische Untersuchungen. Unter günstigen Bedingungen überleben und vermehren sich die meisten pflanzlichen und tierischen Zellen [1]. Dazu werden Zellen in sogenannten Inkubatoren, die einen definierten Lebensraum bereitstellen, kultiviert. In Zellinkubatoren werden Umgebungsparameter wie pH-Wert, Temperatur oder Feuchte gemessen und kontrolliert. Kohlenstoffdioxid $\left(\mathrm{CO}_{2}\right)$ wird genutzt, um den pH-Wert einzustellen und zu puffern. Das gasförmige Kohlendioxid löst sich teilweise in Wasser und bildet Kohlensäure [2]. Dabei werden üblicherweise Konzentrationen von $0-10 \% \mathrm{CO}_{2}$ benötigt. Eine dauerhafte Überwachung der $\mathrm{CO}_{2}$-Konzentration erlaubt eine optimale Einstellung und Regelung der Wachstumsbedingungen innerhalb eines Zellinkubators. Die meisten handelsüblichen Kohlendioxid - Messsysteme messen in einem Messbereich bis maximal $2 \%$ $\mathrm{CO}_{2}$ und werden zur Überwachung der Raumluftqualität eingesetzt. Andere Systeme mit erweitertem Messbereich sind nicht kostengünstig, da sie mit Komponenten, wie IR - LEDs und Photodioden arbeiten. Ziel dieser Entwicklung ist es eine kostengünstige Alternative für den Einsatz in Zellinkubatoren zu realisieren.

Das hier verwendete Messprinzip basiert auf der molekülspezifischen Absorption von IR-aktiven Gasmolekülen. Durch die Absorption von IR-Strahlung durch Gasmoleküle werden Molekülschwingungen 
angeregt [3]. Die kinetische Energie der Schwingungsbewegung des Moleküls entspricht genau der Energie des absorbierten Photons. Diese absorbierte Strahlung ist nach Durchlaufen der Messstrecke nicht mehr im einfallenden Spektrum am Detekor enthalten. Der infrarotoptische Detektor erfasst die Strahlung am Ende der Messstrecke und detektiert die Differenz zum emittierten Spektrum. Ein IR-Filter limitiert das breitbandige Spektrum auf einen Bereich um die zu untersuchende Wellenlänge, bei der Absorption des Messgases stattfindet. Die gemessene Intensität von IR-Strahlung kann in einen Konzentrationswert des absorbierenden Gases umgerechnet werden. Der Anteil der absorbierten Strahlung ist abhängig von der Konzentration des Gases im Probenraum. Durch diese Messmethode lassen sich selektiv Gaskonzentrationen messen, da jedes IR-aktive Gas einen spezifischen Absorptionsbereich hat. Die Strahlung wird im hier vorgestellten System mit einem zweifachen pyroelektrischen Detektor detektiert. Auf dem Dualdetektor sind zwei verschiedene IR-Filter aufgebracht. Als IR-Quelle wird ein thermischer Emitter verwendet, der als annähernd Planck'scher Strahler Infrarotstrahlung in einem breiten Spektrum emittiert.

\section{Aufbau des Gasmesssystems}

Das Messsystem arbeitet mit dem Prinzip der Filterfotometrie bei dem eine Strahlungsquelle infrarotes Licht in eine Messkammer emittiert. Das einfallende Spektrum am Detektor wird mit dem emittierten Spektrum verglichen.

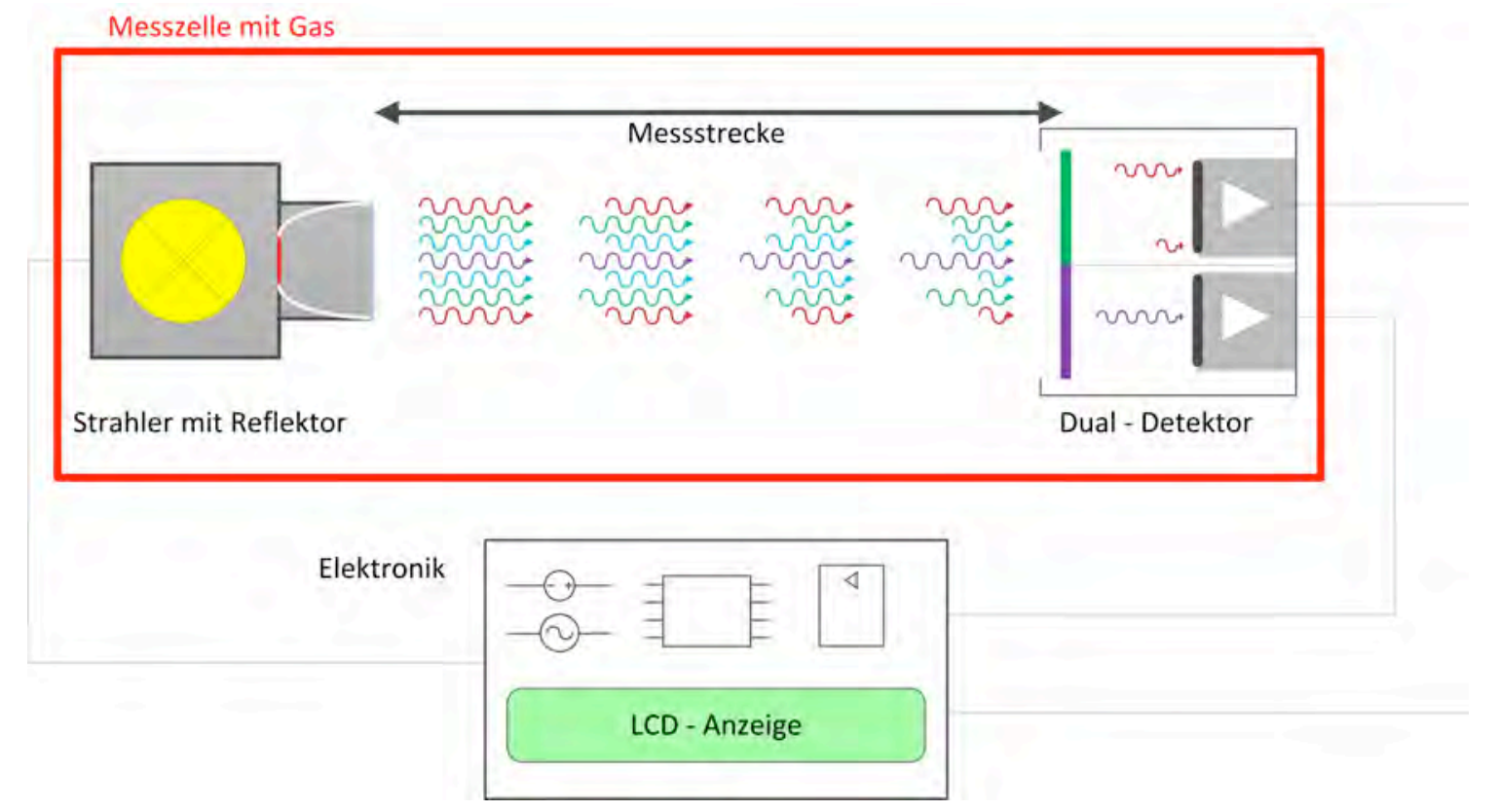

Abbildung 1: Prinzip des vorgestellten Messsystems als Filterfotometer. Der IR-Emitter mit Reflektorgehäuse und der Dual-Pyrodetektor befinden sich in der Gasmesszelle. Die Elektronik zur Messwertverarbeitung und Ansteuerung der Komponenten ist außerhalb der Messkammer.

Ein thermischer Emitter (Intex MIRL17-900) wird mit einer Frequenz von $7 \mathrm{~Hz}$ moduliert, da der im Messsystem verwendete pyroelektrische Detetkor nur auf Strahlungsänderungen reagiert. Der pyroelektrische Dualdetektor (Infratec LIM-262-EH [4]) mit zwei verschiedenen Filterfenstern detektiert bei zwei unterschiedlichen Zentralwellenlängen (CWL). Bei CWL $=4,45 \mu \mathrm{m}$ wird die Absorption aufgrund der vorherrschenden $\mathrm{CO}_{2}$-Konzentration und bei $\mathrm{CWL}=3,95 \mu \mathrm{m}$ wird der Referenzkanal ohne 
spezifische Absorption gemessen. Durch die Division des ermittelten $\mathrm{CO}_{2}-$ Messwertes durch den Referenzwert können Driftverhalten oder Strahleremissionsschwankungen eliminiert werden. Die molekülspezifische Absorption von $\mathrm{CO}_{2}$ liegt hauptsächlich bei einer Zentralwellenlänge von 4,3 $\mu \mathrm{m}$. Die Messung erfolgt versetzt dazu bei einem sekundären Maximum, da die zentralen Absorptionslinien bei der gegebenen Messstrecke schon früh in Sättigung gehen (siehe Abb. 2). Die Messstrecke beträgt $6,25 \mathrm{~cm}$ und kann nicht beliebig reduziert werden, da durch sie der Messbereich bestimmt wird und die IR-Komponenten einen Mindestabstand benötigen, um nicht thermisch überzusprechen. Für die Installation des Systems und die Definition der Messstrecke wurden mechanische Halterungen auf einer Schiene entworfen und hergestellt (siehe Abb. 4).

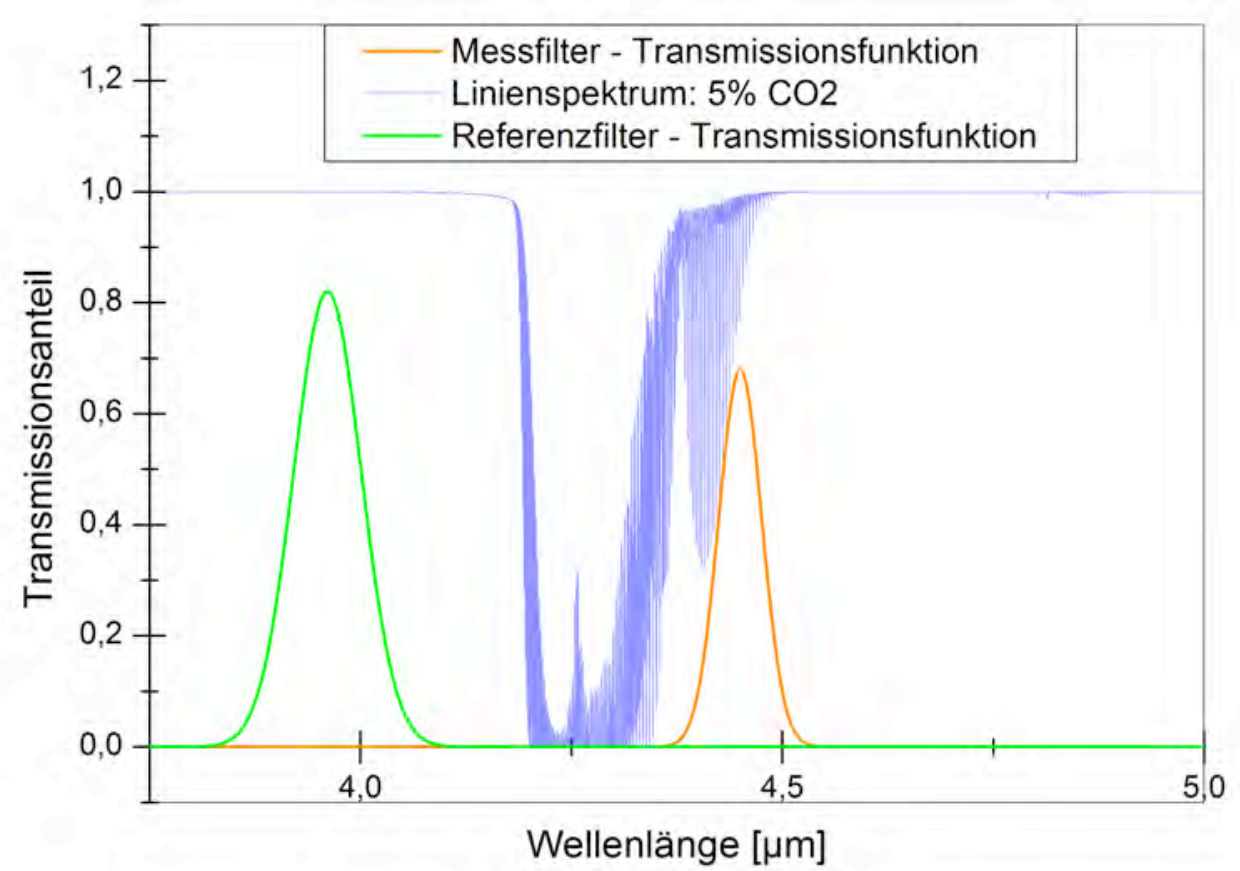

\begin{abstract}
Abbildung 2: Durchlasscharakteristika der verwendeten IR-Filter und Transmissionsspektrum von $5 \% \mathrm{CO}_{2}$ bei einer Messtrecke von 6,25 cm. Die grüne Kurve zeigt den Referenzfilter ohne spezifische Absorption. Die rote Kurve zeigt den Messfilter, der versetzt zur Hauptabsorption von Kohlendioxid liegt, um den gewünschten Messbereich von 0-10 \% zu realisieren.
\end{abstract}

Für eine Signalsbschätzung werden die Durchlasscharakteristika der Filter mit dem Transmissionsanteil des Aufbaus, bei gegebener Messstrecke und $\mathrm{CO}_{2}$-Konzentration multipliziert. Abbildung 3 zeigt die Abschätzung des Signals am Detektor des Messkanals $(C W L=4,45 \mathrm{~cm})$ für $1 \%$ und $10 \% \mathrm{CO}_{2}$. Es ist deutlich der mit der Konzentration steigende Anteil der Absorption zu erkennen, welcher die Änderung des Messsignals hervorruft. Das Integral der in Abbildung 3 ermittelten Funktionen entspricht der Intensität der IR-Strahlung am Detetkor des Messkanals. Die Intensität des Referenzsignals ist idealerweise konstant und beeinflusst die Signaländerung aufgrund molekülspezifischer IR-Absorption nicht. Das resultierende Intensitätsverhältnis aus Mess- und Referenzkanal lässt sich in eine spezifische Kohlendioxidkonzentration umrechnen. 

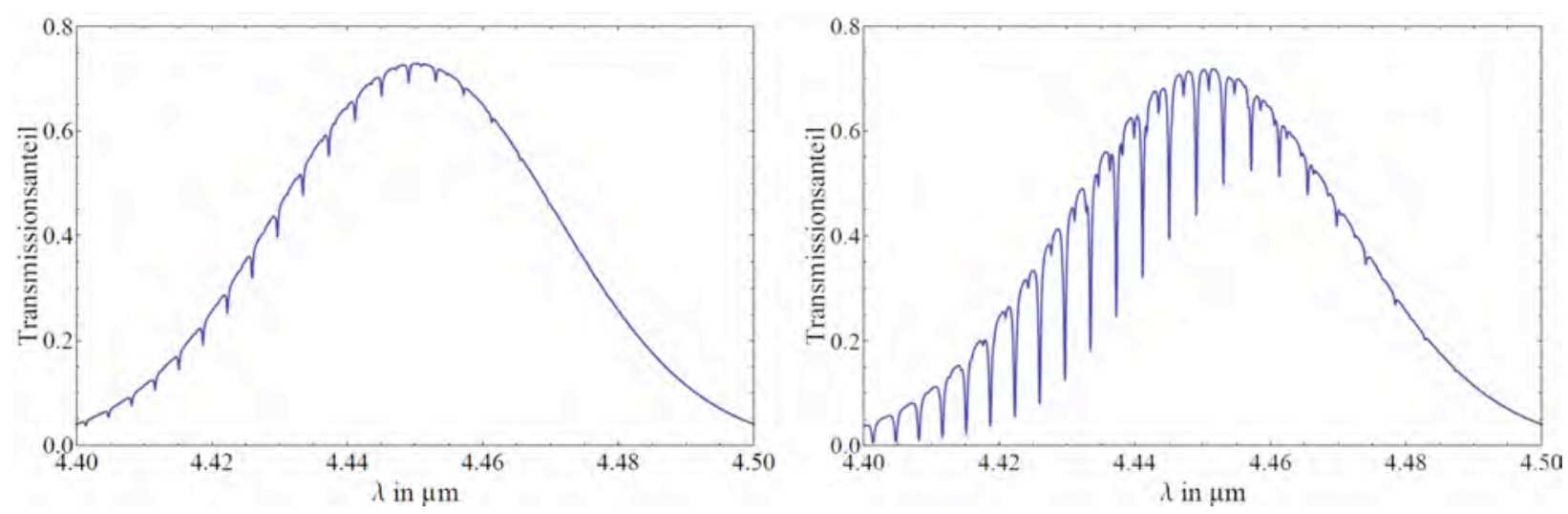

Abbildung 3: Analytische Abschätzung der Intensität des Detektorsignals des Messkanals für verschiedene Kohlendioxidkonzentrationen bei der gegebenen Messstrecke $(6,25 \mathrm{~cm})$ : Im linken Bild ist die Abschätzung für $1 \% \mathrm{CO}_{2}$ und rechts für $10 \% \mathrm{CO}_{2}$ durchgeführt.

\section{Energieversorgung und Signalauswertung}

Für die Ansteuerung und Versorgung der IR-Komponenten, die analoge Verarbeitung des Messsignals, die Digitalisierung und Berechnung eines Konzentrationswertes sowie die Messwertanzeige wurde eine geeignete Elektronik entworfen. Alle elektronischen Komponenten wurden auf einer Platine realisiert.

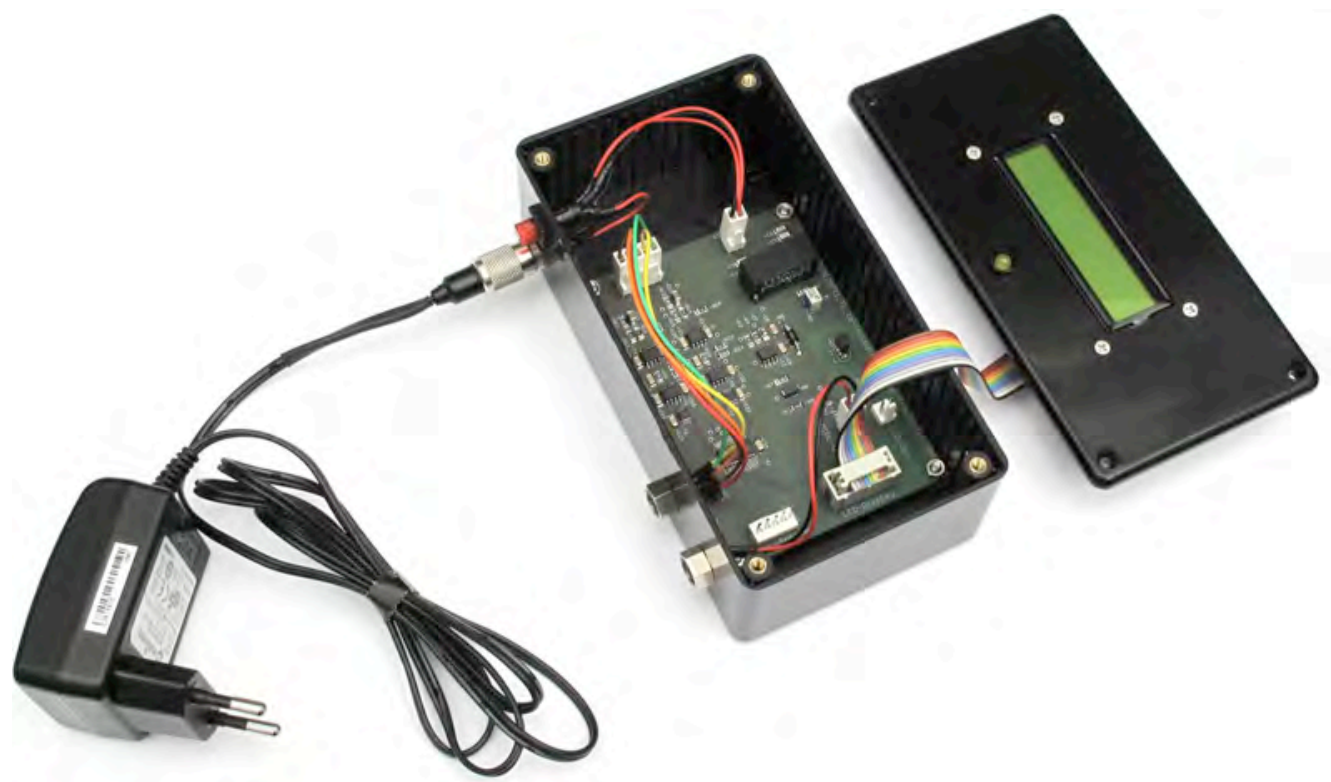

Abbildung 4: Systemelektronik des entwickelten Messsystems zur Ansteuerung der Komponenten und der Messwertverarbeitung. Im Deckel des Gehäuses ist eine LCD-Anzeige zur Visualisierung des Messwertes integriert. Die Spannungsversorgung des Gesamtsystems erfolgt über ein Steckernetzteil.

Die Versorgung des Gesamtsystems erfolgt durch ein Steckernetzteil. Für die Ansteuerung und Modulation des Strahlers wurde eine Timerschaltung mit einem NE555 - Baustein aufgebaut. Die bipolare Versorgung des Detektors wurde mit Hilfe eines DC/DC-Wandlers (Traco TMR 3-1221 WI) 
realisiert. Eine sensornahe Signalauswertung ist unempfindlicher gegenüber elektrischen Störungen. Der Signal-Rausch-Abstand verbessert sich und die Zuverlässigkeit steigt [5]. Eine analoge Bandpassfilterung und Verstärkung der Detektorsignale wurden ebenso, wie die Analog-Digital-Wandlung, in zwei parallelen Zweigen implementiert. Die Wechselsignale der beiden Detektorkanäle werden durch einen Offset in den positiven Messwertbereich verschoben, um die Analog-Digital-Wandlung zu vereinfachen. Die Digitalisierung der Messdaten erfolgt durch zwei 16-bit Sigma-Delta Analog-Digitalwandler (AD7790). Die digitalisierten Daten werden per serielle Schnittstelle an einen mit $24 \mathrm{MHz}$ getakteten Mikrocontroller (Cypress PSoC CY8C29466) gesendet. Daraufhin erfolgt die digitale Messwertverarbeitung im Mikrocontroller. Zunächst werden die Daten digital mit einem Medianfilter gefiltert. Dieser nichtlineare Filter glättet das Messsignal und eliminiert Peaks und Störungen der Messwertaufnahme. Im nächsten Verarbeitungsschritt werden Minima und Maxima des Wechselsignals extrahiert. Die Amplitudendifferenz der Extrema gibt Information über den Anteil der Absorption des infraroten Spektrums. Zuletzt werden die Amplitudeninformationen des Mess- und Referenzkanals dividiert. Diese Methode der Datenanalyse reduziert den Rechenaufwand im Mikrocontroller erheblich und beschleunigt die Messwertverarbeitung. Dadurch können mehr Messwerte verarbeitet werden, was bei typischerweise stark verrauschten Infrarotsignalen eine Verbesserung der Sensorgenauigkeit bedeutet. Abbildung 4 zeigt das geöffnete Elektronikgehäuse mit integrierter Platine, Steckernetzteil zur Energieversorgung und LCD Messwertanzeige. Abbildung 5 zeigt das Gesamtsystem. Auf der Messaufnehmerschiene sind die optischen Messkomponenten montiert. Die im Gehäuse integrierte Elektronik versorgt die Komponenten und verarbeitet die Messdaten zu einem Konzentrationswert.

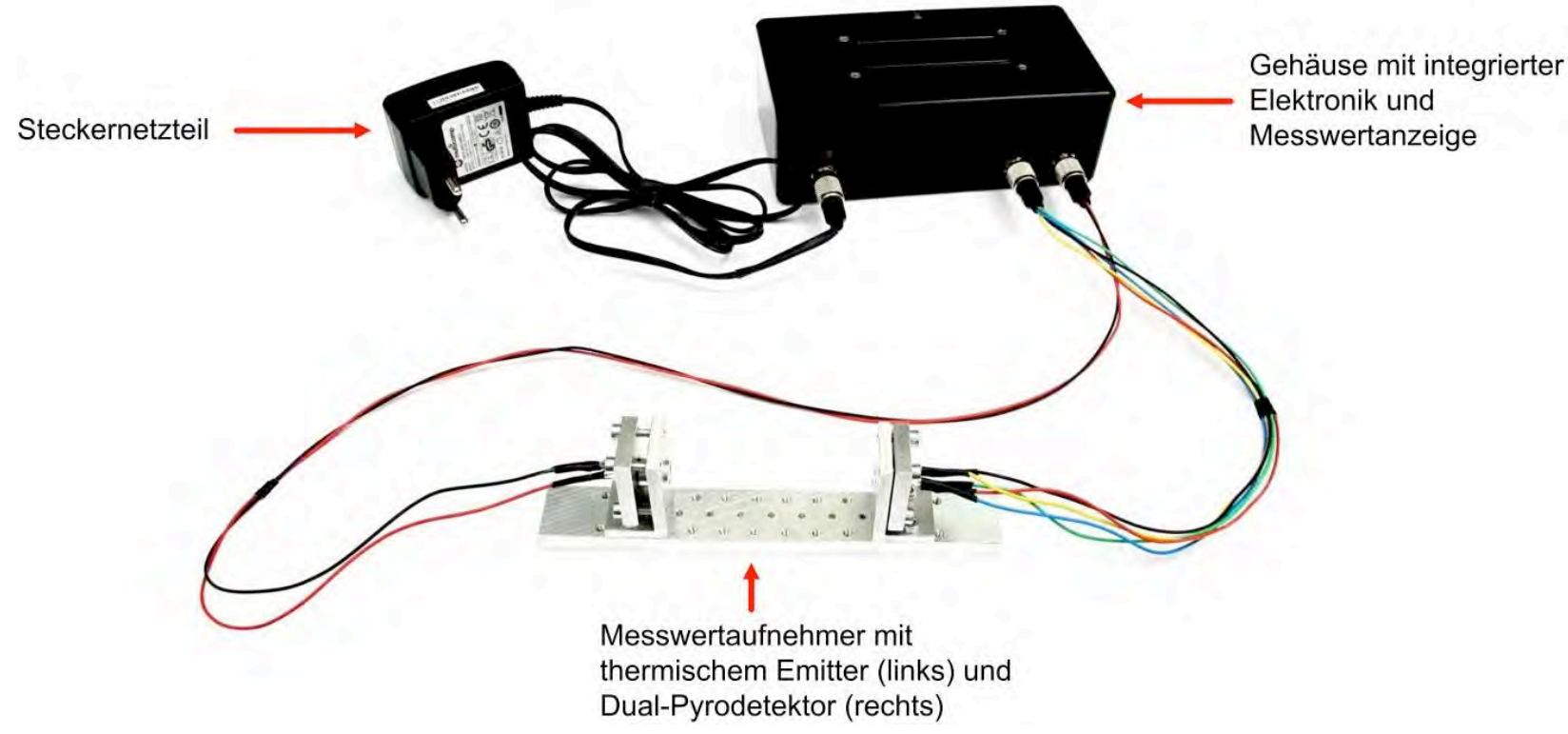

Abbildung 5: Gesamtsystem mit Messaufnehmerschiene, die sich innerhalb des Inkubators befindet. Die Elektronik befindet sich außerhalb des Inkubators.

\section{Experimentelle Ergebnisse}

Das Messsystem wurde bei verschiedenen $\mathrm{CO}_{2}$ - Konzentrationen untersucht. Abbildung 6 zeigt eine Messung in Stufen von $0,5 \% \mathrm{CO}_{2}$ über dem gesamten Messbereich. Die gezeigten Messwerte sind 
einheitenlose Werte des Microcontrollers nach der Digitalsierung. Sie werden nach der spezifischen Kalibration im Zielinkubator in eine $\mathrm{CO}_{2}-$ Konzentration umgerechnet. Das Signal verhält sich nichtlinear, wie es aufgrund der Nichtlinearität der Absorption mit steigender Konzentration des absorbierenden Gases erwartet wird. Die Messung zeigt, dass mit dem entwickelten System der komplette anvisierte Messbereich abgedeckt werden kann. Mit steigender Konzentration sinkt die Auflösung des Systems leicht ab. Eine Messbereichserweiterung ist mit einfacher Modifikation der Absorptionsstrecke möglich. Die Konzentrationsangaben beziehen sich auf die Zusammensetzung des Gases, das in die Messbox eingeleitet wird. Die Gaskonzentration im Volumen nähert sich mit der Zeit exponentiell an die Zusammensetzung des zuströmenden Gases an. Deshalb sind die Stufen der Konzentrationseinstellung in der Messkurve nicht sichtbar.

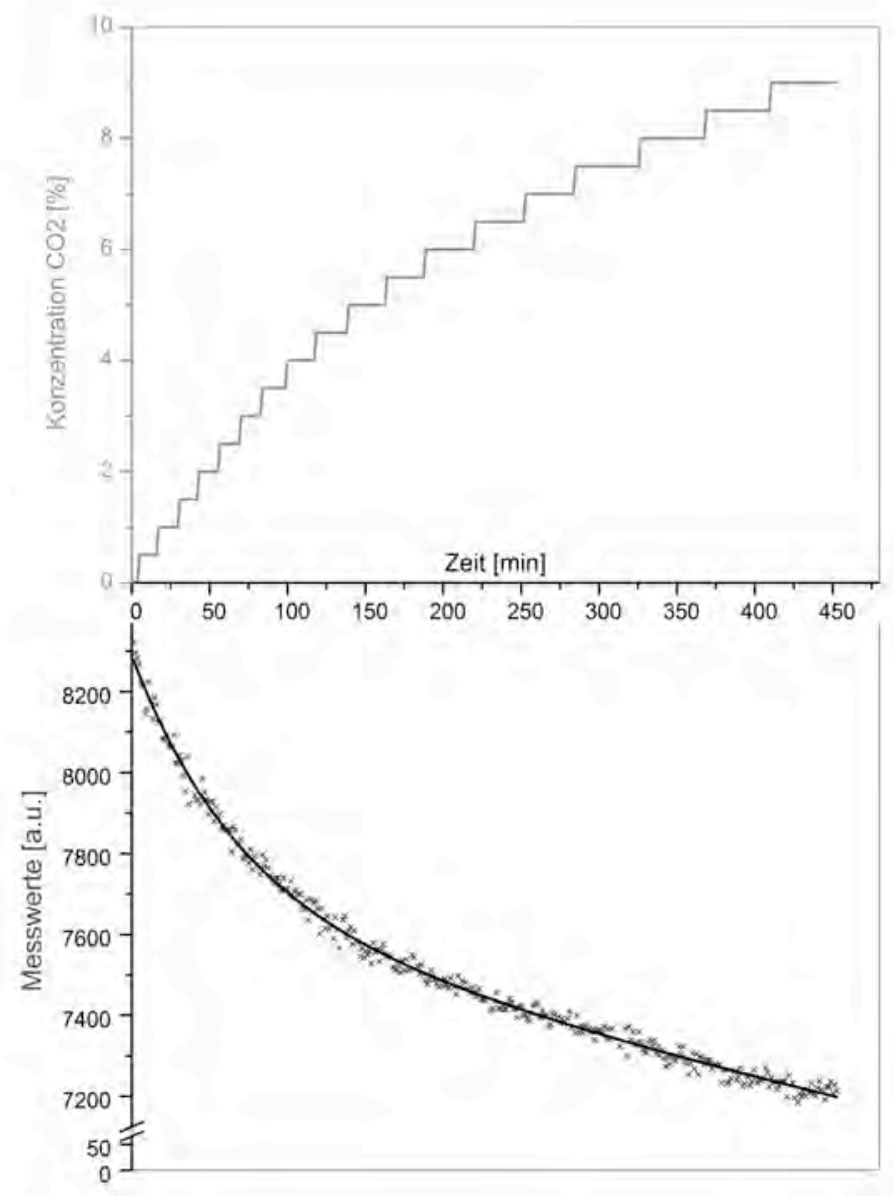

Abbildung 6: Messung in 0,5\%-Konzentrationsstufen von $\mathrm{CO}_{2}$ über den gesamten Messbereich des Systems von 0-10\%. Die Messwerte sind einheitenlose, digitale Werte im Mikrocontroller und werden nach der Kalibration in Konzentrationswerte umgerechnet.

Um die Auflösung des Messsystems zu untersuchen, wurden verschiedene Messreihen aufgenommen. Dabei wurde die Gaskonzentration in gleichmäßigen Stufen erhöht. Die Schrittweiten der Gaskonzentrationsstufen wurden in jeder Messung verkleinert. Die Messungen wurden um die Mitte des Messbereichs bei $5 \% \quad \mathrm{CO}_{2}$ durchgeführt. Bei jeder Messung wird betrachtet, ob die 
Messwertunterschiede der verschiedenen Gaskonzentrationen groß genug sind, um sie mit dem entwickelten Messsystem aufzulösen. Zwischen zwei Gaskonzentrationen wurde das gesamte Volumen mit reinem Stickstoff also $0 \% \mathrm{CO}_{2}$ gespült. Abbildung 7 zeigt die aufgenommenen Messreihen 1-4. Alle Messungen wurden unter gleichen Messbedingungen in der gleichen Messkammer durchgeführt. Die Dauer, in der zwischen den Konzentrationsstufen mit reinem Stickstoff gespült wurde, variiert zwischen den einzelnen Messreihen. Bei den Konzentrationsstufen von 1,0\% und 0,5\% (Messungen 1 \& 2) sind deutliche Messwertunterschiede zu sehen. Man sieht den exponentiellen Verlauf der gemessenen Werte nach einer Änderung der Gaszusammensetzung aufgrund der Zeit bis zur Einstellung des Gleichgewichts in der Messkammer. In einem Inkubator finden typischerweise nur sehr langsame Konzentrationsänderungen statt.
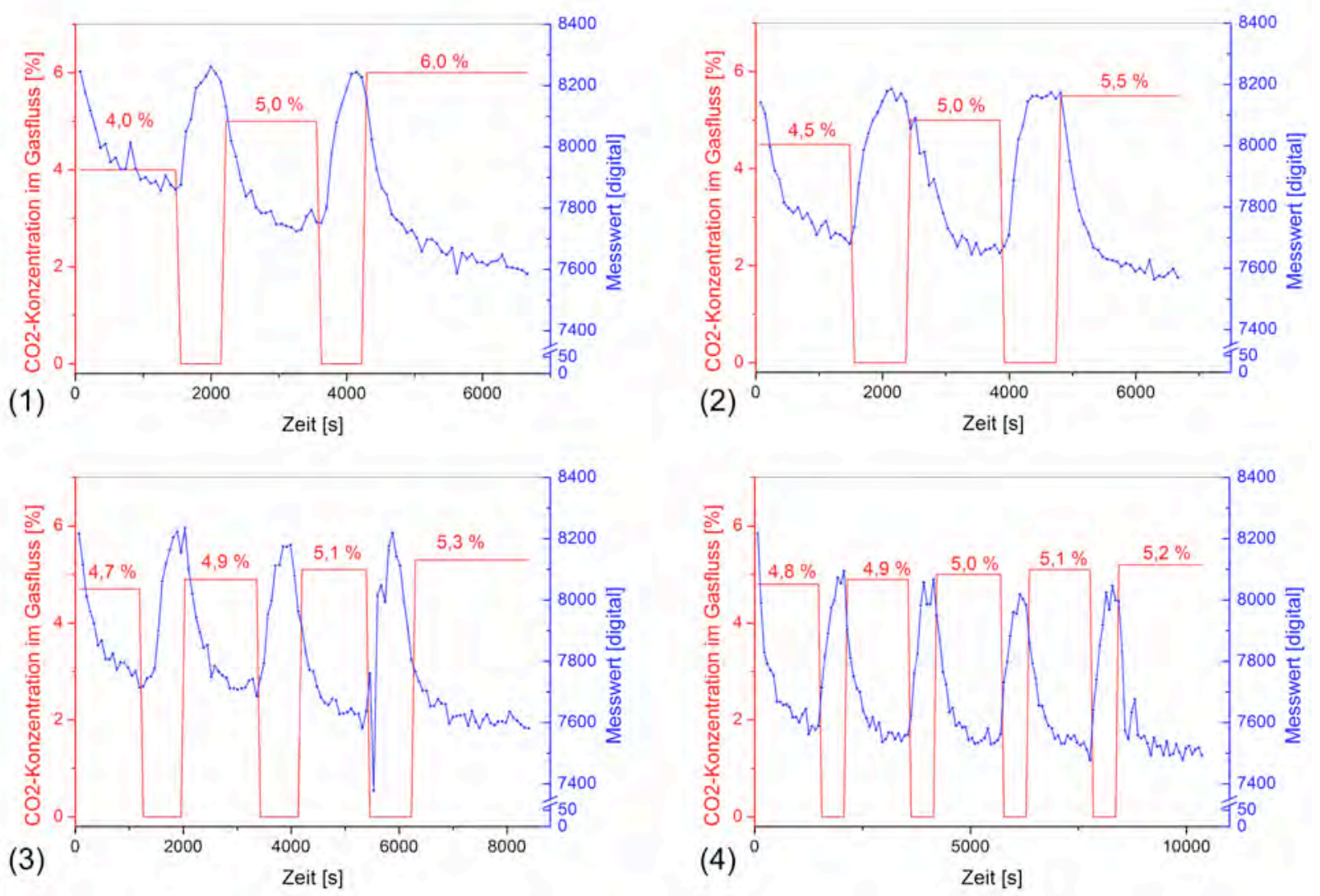

Abbildung 7: Messungen zur Untersuchung der Auflösung des Messsystems. Die Messungen sind in unterschiedlichen Gaskonzentrationsstufen durchgeführt. Zwischen jeder neuen Konzentrationsstufe wurde mit reinem Stickstoff gespült. (1) $1 \%$-Stufen, (2) 0,5\%-Stufen, (3) $0,2 \%-S t u f e n$, (4) 0,1\%-Stufen. Der zeitliche Verlauf der Messwerte ergibt sich aus dem typischerweise großen Volumen eines Zellinkubators, indem sich nur sehr langsame Konzentrationsänderungen einstellen.

Es ist deutlich die Reaktion auf Zusammensetzungsänderungen des Gases innerhalb des Messvolumens zu erkennen. Die 0,2 \%-Stufen-Messung (Messung 3) zeigt ebenfalls signifikante Unterschiede zwischen den einzelnen Konzentrationsstufen. In Messung 4 kann man erkennen, dass auch 0,1\% Unterschied auflösbar sind. Allerdings schwanken die Messwerte bei konstanter Gaszusammensetzung in der gleichen Größenordnung. 


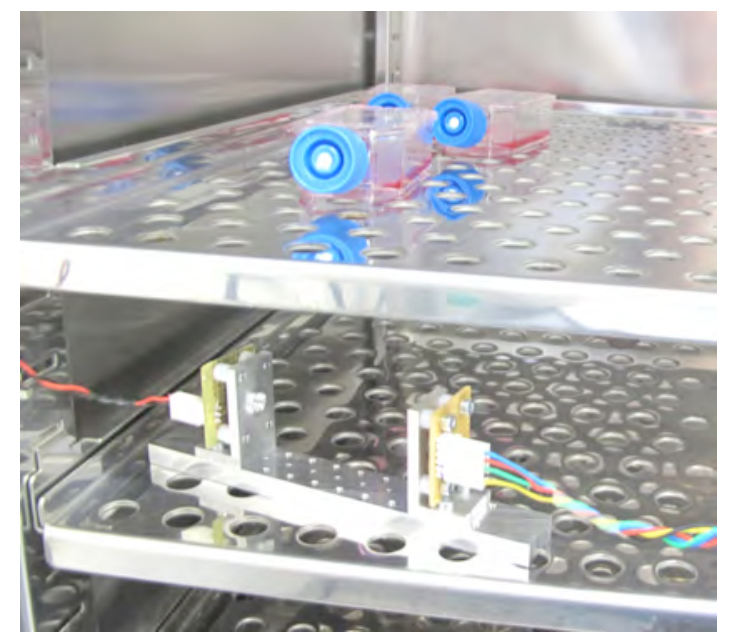

Abbildung 8: Installiertes Messsystem in einem Zellinkubator. Oben sind Zellkulturen zu sehen. Das Messsystem ist beliebig im Inkubator installierbar.

Abbildung 8 zeigt das installierte System in der Anwendung in einem Zellinkubator. Die Messschiene kann beliebig positioniert werden und detektiert die $\mathrm{CO}_{2}$-Konzentration direkt im Probenraum der Zellkulturen.

\section{Zusammenfassung}

Das vorgestellte Gasmesssystem für Zellinkubatoren wurde zur Messung von $\mathrm{CO}_{2}$ in einem Messbereich 0-10 \% in der Gasphase entwickelt. Das System basiert auf einem Filterfotometer und arbeitet mit zwei Messkanälen. Ein Messkanal fungiert als Referenz, wodurch Driftverhalten des Detektors oder des Strahlers keinen Einfluss mehr auf die Messung haben. Das System arbeitet mit einem breitbandigen thermischen Emitter und einem pyroelektrischen Dualdetektor und ist daher kostengünstiger zu fertigen, als alle kommerziell erhältlichen Systeme mit diesem Messbereich. Das System detektiert selektiv und zuverlässig Kohlendioxid in der Gasphase. Die Energieversorgung wurde unabhängig vom Inkubator realisiert. Das entwickelte System eignet sich zur Überwachung der $\mathrm{CO}_{2}$-Konzentration in Zellinkubatoren. Dadurch kann der pH-Wert des Zellkulturmediums kontrolliert werden. Durch diese zeitnahe Überwachung können die Bedingungen innerhalb eines Inkubators geregelt und optimiert werden.

\section{Quellenangabe}

[1] Alberts Bruce und 5 weitere Autoren. Molekularbiologie der Zelle - zweite Auflage. VCH Verlagsgesellschaft, 1994.

[2] S. K. Lower. Carbonate equilibria in natural waters. A Chem1 Reference Text. Environmental Chemistry. Simon Fraser University, 1999.

[3] Günzler Helmut und Hans-Ulrich Gremlich. IR-Spektroskopie, vierte Auflage. WILEY-VCH,Weinheim, 2003.

[4] InfraTec GmbH. Datenblatt: LIM-262. http://www.infratec.de.

[5] Schrüfer E. Elektrische Messtechnik, 7. Auflage. Carl Hanser Verlag, München Wien, 2001. 